\title{
Study on Autonomous and Distributed Time Synchronization Method for formation UAVs
}

\author{
Liu Tao ${ }^{1,2,3, a}$, Hu Yonghui ${ }^{1,2, b}$, Hua Yu ${ }^{1,2, c}$, Wu Meifang ${ }^{1,2,3, d}$ \\ ${ }^{1}$ National Time Service Center /Key Lab of Precision Navigation and Timing ${ }^{2}$ Technology, Chinese \\ Academy of Sciences, Xi'an, Shaanxi China \\ ${ }^{3}$ University of the Chinese Academy of Sciences, Beijing, China \\ aliutao@ntsc.ac.cn, bhyh@ntsc.ac.cn, chy@ntsc.ac.cn, dwumeifang@ntsc.ac.cn
}

Keywords: UAVs. Autonomous. Distributed. Time synchronization.

\begin{abstract}
In order to implement high precision time synchronization autonomously in the absence of any external time source, in this paper, the author introduced the synchronize model of fireflies into UAV formation network, and proposed a kind of distributed time synchronization methods based on broadcasting, and conducted some computer simulation experiments and built one test platform to prove the feasibility of the method and its performance, the results show that the method can effectively achieve time synchronization autonomously without any external time source, and the synchronization accuracy can be achieved about 100us.
\end{abstract}

\section{Introduction}

Unmanned aerial vehicles (UAV) has an irreplaceable role in modern war, the high-precision time synchronization is a key technology to achieve multi-UAVs formation flight, cooperative reconnaissance and collaborative attack. However, the existing pattern that depending GNSS satellite navigation system, or ground control station for time synchronization, its independent properties are subject to certain restricted, especially in wartime, there is a serious security risk strategy. At the same time, for the distributed autonomous UAVs, in the mode of autonomous aviation, there is no fixed location or known the exact coordinates of the reference station, nor accurate external time reference, for most cooperative task, the relative position and relative clock face between the UAVs is more important than the absolute difference between the UAV and the UTC time. So, how to achieve the time synchronization with high precision for all UAVs in the whole network completely and independently in the absence of any external time source is imminent.

For the distributed architecture such as large-scale UAVs formation, the traditional master-slave time synchronization method is no longer applicable, and the firefly synchronization model which ancient origin and had been studied in biology, chemistry and mathematics fields provides a new way of thinking to solve the problem of distributed time synchronization. In this paper, the author introduced the model of synchronization for the fireflies in biology into UAV formation network, and proposed a distributed time synchronization method based broadcasting, make use of the existing communication links between UAVs, each UAV broadcasts its current time information, after its corresponding neighbor nodes receiving the information, these received information do simple arithmetic average, the average value as the clock tick for the next time then broadcast again, this process is repeated several times, all the nodes in the network will ultimately lead to an identical clock reaches on average, that implement the distributed time synchronous for the whole formation network.

\section{The UAV Structure}

The Structure of UAV Formation. Because the working environment for UAVs formation and self-organization network is similar to the Ad-hoc network, is completely self-organization and distributed architecture, therefore, this article will build UAV formation self-organizing network based on a system of the Ad-hoc network. 
The UAV formation is composed of a few UAVs as a network and can communicate with each other, each UAV acts as a node in the network, the way to form a network with no central node, the position between each node is equal. Taking one network with five UAVs as an example, the network model is shown in Fig 1.

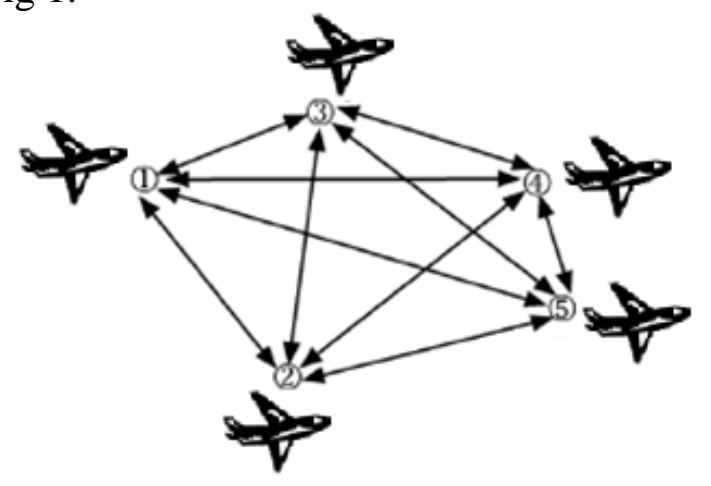

Fig.1: The Structure of UAVs formation

The five nodes can communicate with each other, and there is no central node between five nodes, when a node has been shot down or lost for other reasons, the remaining nodes can still form a network, keeping communications and do other tasks, that is, within a network, the lost of the nodes can not affect the reconstruction of the network. At the same time, each node in the network can use their assigned a particular frequency and a spreading code to spread spectrum modulation, and sends a message to other neighbors, in addition, this process is a broadcasting-style, achieved a single point to multi-point sends multiple access. In this paper, the author build a full-connected network, that is, in the network, one node sends a message, all the remaining nodes are able to receive it, to ensure the communication is full coverage of the network.

The Communication of UAV Formation. The UAV formation flight is kind of multi-user and short-range wireless communication, requirements for communication systems with high transmission speed, low-power, anti-interference ability, high system capacity, multi-site resolution ability and other characteristics, and the Ultra Wide Band(UWB) wireless communication technology just has the above advantages, is very suitable for the establishment of links, as well as data transmission and other monitoring and control functions among the UAVs.

The UWB communication standard is different from the traditional, it transmits information is by loading data into a very short duration (typically picoseconds to nanoseconds), low duty cycle pulse signals, it no longer has the concept of the intermediate frequency(IF) and the Radio frequency(RF), the transmitting and receiving of the signal will not undergo the like mixers, carrier modulation and demodulation process, the transmitting and receiving equipment is relatively simple, and another particular importance is that the signal propagation delay is very small.

In summary, in this article, the author make use of the UWB communication systems in UAV formation, taking the UWB signals as an information carrier, UAV formation UWB communication system as an information carrier, and gotten help from the characteristics of having a minimal transmission delay, combining the mathematical model of fireflies and achieve the distributed time synchronization for UAV formation.

\section{Mathematical model}

Scientists have often looked to nature for inspiration. Swarms of fireflies stretching for miles can pulse in perfect unison, all without centralized control or perfect individuals. This phenomenon provides us a new thinking to obtain time synchronization in distributed formation UAVs. In biological systems distributed synchronization is commonly modeled using the theory of coupled oscillators. For fireflies, an oscillator represents the internal clock dictating when to flash, and upon reception of a pulse from other oscillators, this clock is adjusted. Over time, synchronization emerges, i.e. pulses of different oscillators are transmitted simultaneously [2]. A theoretical framework for the convergence to synchrony in fully-connected mesh networks was proposed by 
Mirollo and Strogatz, before introducing the synchronization strategy adapted to formation UAVs, the mathematical model of Mirollo and Strogatz should be presented firstly as follows.

The internal clock of a firefly, which dictates when a flash is emitted, is modeled as an oscillator, and the phase of this oscillator is modified upon reception of an external flash. In the remainder, we focus on integrate-and-fire oscillators, which are also termed "pulse-coupled oscillators" . They interact through discrete events each time they complete an oscillation. The interaction takes the form of a pulse that is perceived by neighboring oscillators [2].

To demonstrate that synchrony is always achieved independently of initial conditions, each node has an internal time or phase $t$, it is described by a phase function $\phi_{i}$, which starts at zero and linearly increments from 0 to a phase threshold $\phi_{\text {th }}$ and periodically "fires" every T seconds. At this point the node "fires" (in the case of firefly, flashes), in a wireless communication system, the "fire" means transmitting a synchronization signal, then resets its phase to 0 , and begin to linearly increments. So, we can obtain the following equation:

$$
\frac{d \phi_{i}(t)}{d t}=\frac{\phi_{t h}}{T}
$$

In the absence of any input from neighbors, it will naturally oscillate and fire with a period T. Fig. 2(a) plots the evolution of the phase function during one period when the oscillator is isolated. When coupled to others, the node is receptive to the pulses of its neighbors. Coupling between nodes is considered instantaneous, and when a node $\mathrm{j}(1 \leqslant \mathrm{j} \leqslant \mathrm{N})$ fires at $t=\tau_{j}$, i.e. $\phi_{j}\left(\tau_{j}\right)=\phi_{t h}$, all nodes adjust their phase function as follows:

$$
\phi_{j}\left(\tau_{j}\right)=\phi_{t h} \Rightarrow\left\{\begin{array}{l}
\phi_{j}\left(\tau_{j}\right)=0 \\
\phi_{i}\left(\tau_{j}\right)=\phi_{i}\left(\tau_{j}\right)+\Delta \phi\left(\phi_{i}\left(\tau_{j}\right)\right) \quad i \neq j
\end{array}\right.
$$

The received pulse causes the oscillator to fire early. The parameter $\Delta \phi\left(\phi_{i}\left(\tau_{j}\right)\right)$ in Eq. (2) is the phase increment. By appropriate selection of $\Delta \phi$, a system of $\mathrm{N}$ identical oscillators forming a fully-meshed network is able to synchronize their firing instants within a few periods [2]. Fig. 2(b) plots the time evolution of the phase when receiving a pulse.
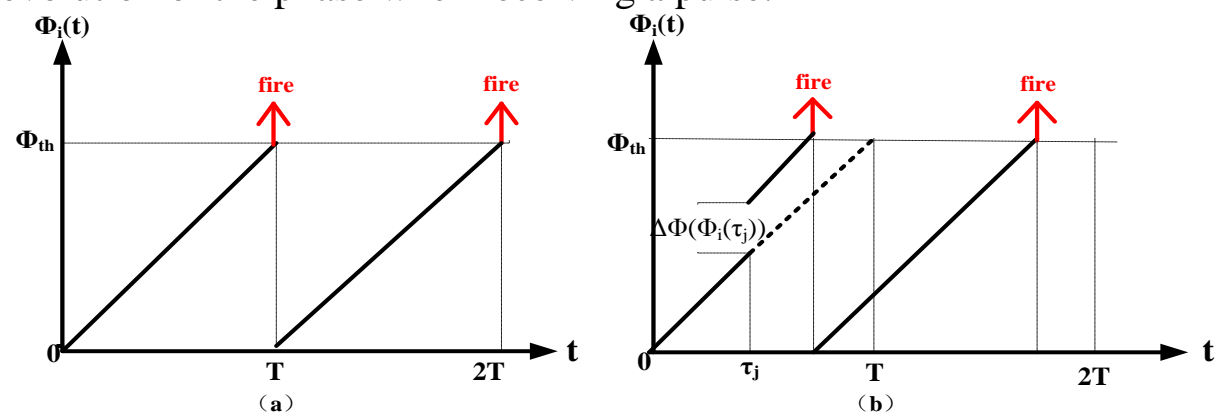

Fig.2: The time evolution of the phase function

\section{Time synchronization in UAV formation}

Introducing the above model into the UAV formation network, each UAV corresponds to a node of the model, and the clock of UAV corresponds the phase information of the node in the model, the specific time synchronization method in UAV formation is as follows:

When a node sends clock information to its neighbor nodes, all the nodes in its broadcast domain are able to receive this information, similarly, a node receives the clock information, also can receive many sets of the clock information on other neighboring nodes. For each node in the network connectivity, $i=1,2, \cdots, N$, having different initial phases, such as $\varphi_{1} \neq \varphi_{2} \neq \cdots \neq \varphi_{n}$, assuming there is no frequency deviation of the nodes in the network, that is to say, each node has the same period, such as $T_{i}=T_{j}, \quad i \neq j, \quad i=1,2, \cdots, n, \quad j=1,2, \cdots, n$. 
Once the phase of a node increase to the threshold, broadcast its current clock information, $\varphi_{i}(n), \quad n=1,2, \cdots, N$; For the receiving node $\mathrm{j}$, may be able to receive $\mathrm{K}$ sets of the clock information at a time, then the node $\mathrm{j}$ will calculating the arithmetic mean of the clock information, that is $\frac{1}{K} \sum_{i=1}^{K} \varphi_{i}(n)$, and put the average value as the clock tick for the next time, there is $\varphi_{j}(n+1)=\frac{1}{K} \sum_{i=1}^{K} \varphi_{i}(n)$, and then the node $\mathrm{j}$ will continue to broadcast the updated clock information to its neighbor nodes.

The above process is repeated, according to the mathematical model, every time the node updates the clock information, correspond to adjust its clock to the mean time, after several times, the clock information of every node will be infinitely close to the mean value of all the nodes, and at some time, all nodes within the network to reach a same clock tick value, that is distributed phase synchronization is achieved throughout the network.

\section{Analysis and Test of Method Performance}

In order to validate the theoretical method presented in the preceding sections, we conducted extensive simulations in MATLAB.

In the simulation, we make use of five nodes, built up such topology as shown in Figure 1, to simplify the simulation, the periods of nodes are discredited to integers that are uniformly distributed in a certain interval, the phase of the nodes is normalized, the initial phase of each node uniformly distributed in the interval $(0,1)$, at the same time, according to our proposed algorithm, after broadcasting the phase information, there will be a delay at the receiving nodes, we assume the random delay is evenly distributed in $(0.001,0.01)$ interval, the vertical axis represents the phase of the nodes, and the horizontal axis represents the synchronization period, the results of the simulation is shown in fig 5 .

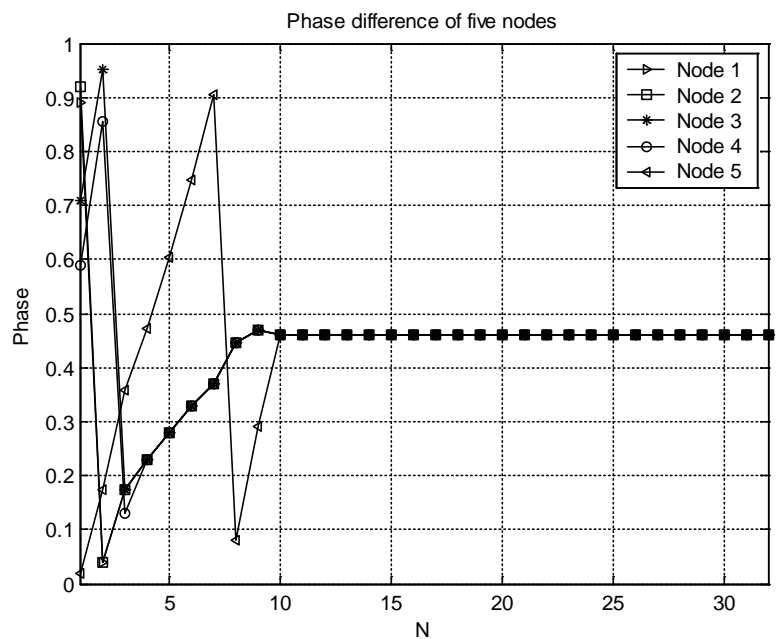

Fig.3: Phase difference of five nodes

As can be seen from the figure, at the start time of the synchronous process $(n=0)$, the phase difference of each node is large, through each synchronization period, the phase difference of each node is reduced, we can see, after 10 cycles(n=10) synchronized, each node to reach a same phase value, and over time $(n \geqslant 10)$, each node in the network has a phase difference of zero, that all network nodes to achieve a phase synchronization.

\section{Implications in UWB test beds and Performance Evaluations}

In this section, we introduce our platform of the test, and including specific test methods. At last, we tested the synchronization accuracy and evaluated the performance for using UWB signal to achieve the distribute time synchronization based on the M\&S model. DV9110M is Wisair 
launched the second generation development board, the synchronization algorithm was implemented analogously to the implementation in DV9110M.Our test environment is made up with five nodes, deployed a topology of all-to-all. In the network, each node is distributed random initial phase, and the nodes were separated by a distance of 10 meters (the program delay about 33 ns). In the test, we used a 16-bit timer to represent the synchronization interval. The timer is configured in CTC mode where the Input Capture Register represents the top value and the period of the node is stored in OCRIA register.

Assuming a nominal oscillator frequency of $8 \mathrm{MHz}$, we decided to set a prescaler of 1024 . As a result we get a granularity of 125000 ticks per second. This should be good enough to achieve a synchronization precision lower than one microseconds. Whenever a node receives a pulse signal, compare the current count in TCNTI with the couter Max value saved in OCRIA, if the threshold is reached, trigger an interrupt, transmission pulse signal, and reset its count to 0; if not, node calculates the phase increment, added to the current count, and the count continues.

When the count reaches $\mathrm{T} / 2$ ( $\mathrm{T}$ is the period) of the node, triggers an interrupt in INT4 pin, all other nodes send back their count value as a set of data, that is, each set of results contains 15 32-bit integer, respectively corresponding to the count value of the counter on each node, calculating the standard deviation of these 15 sets of data, used to measure the results of synchronization errors. Collect multiple sets of data, and calculating the average, then divided by the clock frequency can be synchronized precision. Take one node as an example, synchronization error was collected between the node and the remaining four nodes, and the test data is processed in MATLAB. The results are of Fig.4. The vertical axis represents the synchronization accuracy, the unit is microsecond, the horizontal axis represents the time, and the unit is second.

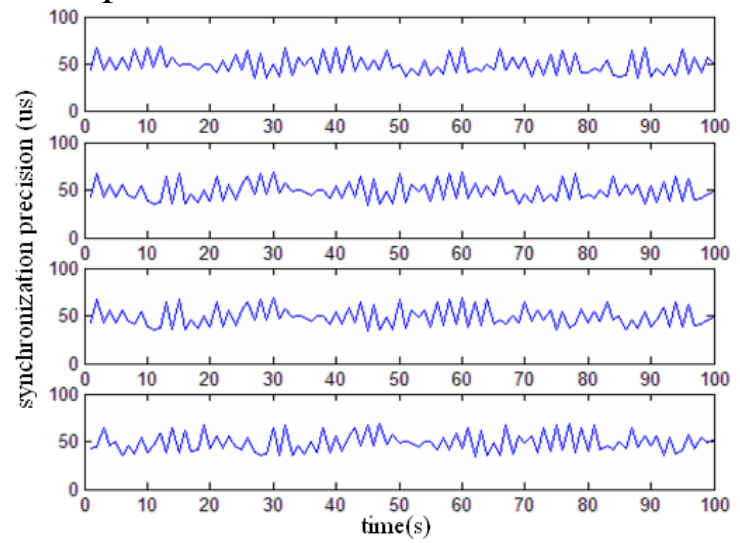

Fig. 4: The test results of synchronization accuracy

The results show that, the synchronization accuracy is about 50 microseconds, at the same time, the accuracy is slightly larger than the theoretical results, and this may be caused by the frequency deviation between the nodes.

\section{Conclusion and future work}

Traditional centralized time synchronization mechanism is applied in large-scale distributed wireless networks, exist serious error accumulation, at the same time, with the complex changes in the network topology, the robustness of the algorithm being seriously challenged. The distributed time synchronization mechanism based on the M\&S model can solve the above problem. However, the inevitable transmission delays restrict the application of the mechanism seriously. In this paper, make use of characteristics that transmission delay is small for UWB signal, compare with conventional signal, through theoretical analysis, we consider that the M\&S model is still suit for UWB signal. To verify our analysis and completion, we built a test platform, and implemented the time synchronization algorithm on the platform. The final test results show that make use of UWB signals, the time synchronization algorithm based on the M\&S model can synchronize multiple nodes indeed, and the synchronization accuracy has strong correlation with the delays of the signal. 
Our future research will focus on the frequency deviation between the nodes, and make further improvement of the synchronization accuracy.

\section{Acknowledgements}

The research work was supported by National Natural Science Foundation of China under Grant No. 11403033.

\section{References}

[1] Alexander Tyrrell, Gunther Auer, Fireflies as Role Models for Synchronization in Ad Hoc Networks, I1-4244-0463-0/06/\$20.0O (C2006 IEEE.

[2] Alexander Tyrrell, Gunther Auer. Biologically Inspired Synchronization for wireless network. Springer-Verlag Berlin Heidelberg 2009.

[3] Daniel Gonzales, John Hollywood, Gina Kingston. Network-Centric Operations Case Study: Air-to-Air Combat With and Without Link 16. National Defense Research Institute, 2005.

[4] King-Yip Cheng, A Distributed Multi-hop Time Synchronization Protocol for Wireless Sensor Networks using Pair-wise Broadcast Synchronization. IEEE Transactions on Wireless Communications, Vol. 8, No. 4, April 2009.

[5] Wei Wu and Tianping Chen. Asymptotic Synchronization for Pulse-Coupled Oscillators with Delayed Excitatory Coupling Is Impossible. Advances in Computational Intell. AISC 61, pp. 45-51.

[6] Y.-W. Hong and A. Scaglione. A scalable synchronization protocol for large scale sensor networks and its applications. IEEE Journal on Selected Areas in Communications. pp. 1085-1099, 2005.

[7] R.C.Qiu, A study of the ultra-wide band wireless Propagation channel and optimum UWB receiver design, IEEE J.Select. Aleas Commun, vol.20， PP.1628-1636, 2002 